\title{
Good cause for celebration
}

\section{Next week's conference to celebrate the thirtieth anniversary of the discovery of the structure of DNA in Boston is more than an exercise in hagiography.}

WHAT more can there be to say by way of celebration of the publication of the structure of DNA (J. D. Watson and F. H. C. Crick Nature 171,$737 ; 1953)$ ? That is a proper question. Nature held a conference on the theme in Cambridge (England) earlier this year. Why now another (next week) in Boston, Massachusetts? Is there not a danger that the topic will be done to death? Or that endless rounds of celebrations will endow particular events with a spurious importance, distracting people's attention from the simple truth that even the most striking discoveries are as much the products as the determinants of the intellectual climate of their times?

Of course. And nobody has an interest in making more of a thirty-year-old discovery than the circumstances warrant. But the circumstances are remarkable. Rarely can there have been an occasion when the effect of a discovery has been to provide so quickly a persuasive answer to a question in the forefront of many people's minds: what is the genetic material, and how does it work?

Newton's theory of gravitation was a welcome surprise to some of his contempories, but a matter of indifference to most of them. Darwin's evolution, widely recognized as important, had to be rammed down the throats of many of his contemporaries. The significance of Planck's quantum went unrecognized (even by Planck). Einstein's special relativity, even with its echoes of earlier work by Lorentz and Poincaré, made sense to some people but nonsense to others. Even the development of the quantum theory in 1925 and 1926 , which predicated the most radical revision of the conception of physical reality (not yet complete) but which also made possible what now passes for physical science (not to mention the electronics industry), was blunted, at the time, by confusion - Schrödinger's sneaking hope that wave mechanics would reconcile the inconsistencies of classical mechanics, the lack of a language (whose grammar Dirac provided), conceptual disputes (which persist) and sheer complexity.

The effect of the publication of the correct structure for DNA was much more immediate. If major discoveries are keys to locked doors, this key has been quickly fitted to its lock and the door has swung open on well-lubricated hinges. Much of the pleasureable excitement of the past thirty years has been the way in which simple expectations of nature have been repeatedly confirmed. If DNA is the genetic material, there must be a biochemical mechanism by which it replicates faithfully (J. D. Watson and F. H. C. Crick Nature 171, 964; 1953), and, indeed, there is (Kornberg, 1956, M. Meselson and F. W. Stahl Proc. natn. Acad. Sci. U.S. A. 44, 671; 1958). If genetic information rests in the arrangement of consecutive nucleotides along the length of DNA molecules, there must be a code which, at its simplest, will be a triple code; right again (F. H. C. Crick et al. Nature 192, 1227; 1961). And there must be some kind of messenger for carrying genetic information from the nucleus to the cytoplasm of a cell (S. Brenner, F. Jacob and M. Meselson Nature 190, 576; 1961) . . . and so on. Along the way, inevitably, there have been surprises, reverse transcriptase for example. And nothing of what has been accomplished in the past three decades would have been possible without the literally marvellous techniques that have been developed and which have led to the deliberate manipulation of genes and their elements. (See page 189 for the first functional synthetic chromosome.) Can such a torrent of excitement be over-celebrated?

To whom, or what, should go the credit? The two obvious recipients are by now sated with congratulation, but it is easy to forget that, thirty years ago, confusion about the genetic mechanism was not much less serious than the confusion now about the strong nuclear interactions (is there a top quark and all that?). How otherwise could Avery's early experiments, in retrospect a convincing demonstration that DNA can transform the genetic character of organisms, have made so slight a mark on the climate of opinion? (The Royal Society awarded Avery its Copley Medal, showing that the importance of his work was recognized, but the citation referred to the "introduction of chemical methods in immunology", showing that its meaning was not.) Part of the trouble was the hankering after the notion that proteins are the genetic material, which no doubt is why Chargaff's demonstration of the constancy of the nucleotide ratios (A to $T$ and $C$ to $G$ ) was at first regarded as a puzzle, not part of an explanation. Watson and Crick's success was twofold: their model was simple, but it embodied not merely a means for encoding genetic information but the means for generating the mechanism of its own replication.

What happened in the decade after 1953 is too easily forgotten. The door may have swung open, but there were still many who suspected it was the wrong door. Biologists were reading the wrong books. Schrödinger's What is Life? is much quoted as an antecedent of the double helix. Von Neuman's work, now flourishing under the banner of "cellular automata" (see S. Wolfram, Rev. mod. Phys. 55, 601; 1983) would have been a more robust guide to the logical requirements of self-replicating systems. In the event, it was Crick, egged on by Gamov's speculations (Nature 173, 318; 1954) who improvised the logic characterizing the genetic code before its existence had been demonstrated. It remains a triumph of imagination over the inconsistencies and gaps in data that he and Brenner should have postulated the existence of adaptor molecules, now called transfer-RNA, before they were in due course found. The door would have been left to swing open in the wind had it not been for the proselytizing zeal in the 1950s of a small band of people devoted to the principle that even biology is not so complicated that it cannot be understood.

None of this should be mistaken for an arrogant supposition that there are no problems left. Plainly that is far from true. Even the mechanisms of DNA replication and transcription are not yet known in sufficient detail to satisfy normal and practical curiosity, let along the purists. The need also to understand how messenger molecules are processed, which is not yet possible, has become apparent only in the past decade. The control of genes in higher organisms is far from understood. Little worth knowing is known in molecular terms about the process of cellular differentiation. Molecular biology has so far contributed to the problem of the origin of life principally the conviction that the problem will prove soluble. That a biotechnology industry is now being built is not surprising (which is not to demean the ingenuity of those who saw how that might be done), but the tasks now being attempted will almost certainly be swamped by those not yet imagined. What has changed for certain, and permanently, is the style of biological research. Asking the right questions (those which can be answered simply) is the objective. Simplifying experiments have come to complement observation. The expectation that answers to the right questions will be generally useful has been widely sustained in the past thirty years. That is the cause for celebration.

John Maddox 